\title{
An urban-rural comparison of the prevalence of the metabolic syndrome in Eastern China
}

\author{
Xiaoping Weng ${ }^{1}$, Youxue Liu', Jiemin $\mathrm{Ma}^{2}$, Wenjuan Wang ${ }^{3}$, Gonghuan Yang ${ }^{2}$ \\ and Benjamin Caballero ${ }^{1, *}$ \\ ${ }^{1}$ Center for Human Nutrition, Johns Hopkins Bloomberg School of Public Health, Baltimore, MD 21205, USA: \\ ${ }^{2}$ Chinese Academy of Medical Science, Beijing, China: ${ }^{3}$ Chinese Center for Disease Control and Prevention, China
}

Submitted 8 July 2005: Accepted 3 April 2006

\begin{abstract}
Objective: To assess the impact of urbanisation on the prevalence of the metabolic syndrome in Chinese adults.

Design: As part of a community-based cross-sectional survey conducted in 2002, a sample from rural and urban populations in East China was obtained. The metabolic syndrome is defined by the National Cholesterol Education Program Adult Treatment Panel III criteria (ATP III) and the modified ATP III, which recommended a lower waist circumference cut-off for Asians.

Setting: Field sites in Jiangxi and Anhui provinces and the Jing'an District of Shanghai, China.

Subjects: A total of 529 non-pregnant, non-lactating urban and rural adults, aged 2064 years without diagnosed diabetes.

Results: Dwelling in urban areas was associated with higher dietary fat intake and slightly lower total energy intake, and with significantly lower occupational physical activity. Using the ATP III criteria, the prevalence of the metabolic syndrome was significantly higher for urban than rural men $(12.7$ vs. $1.7 \%, P<0.001)$, and was similar between urban and rural women (10.1 vs. 9.7\%, $P=0.17)$. These urban-rural differences were greatly enhanced when the modified ATP III criteria for the syndrome were used, for men (34.3 vs. $2.7 \%, P<0.01)$ and women (24.1 vs. 11.4\%, $P=0.07$ ). The Asian waist circumference cut-offs ( 90 and $80 \mathrm{~cm}$ for men and women, respectively) had a better combination of sensitivity and specificity in identifying other metabolic disorders, which included high glucose, high blood pressure, high triglycerides and low high-density lipoprotein cholesterol, for this population. Conclusion: For the Chinese population, urban dwelling was associated with higher prevalence of the metabolic syndrome, especially in men.
\end{abstract}

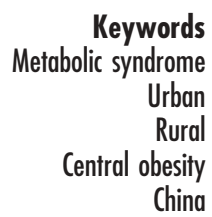

Substantial socio-economic and demographic changes have taken place in China over the past two decades. Since 1982, the urban population increased from 21 to $36 \%{ }^{1}$. A number of studies have shown that urban dwelling is associated with an increase in adverse health risk behaviours and lifestyle changes ${ }^{2-4}$. In the case of China, these demographic changes have been temporally associated with a rapid rise in the prevalence of cardiovascular disease, which is now the leading cause of death ${ }^{2,5}$.

The metabolic syndrome, also called insulin resistance syndrome or syndrome $\mathrm{X}$, is a cluster of metabolic disorders that includes central obesity, insulin resistance, dyslipidaemia and hypertension ${ }^{6}$. It is a well-recognised risk factor for type 2 diabetes mellitus ${ }^{7-9}$ and cardiovascular disease $(\mathrm{CVD})^{10-12}$.

There are no uniform criteria for diagnosis of the metabolic syndrome. The widely accepted working definition, proposed by the National Cholesterol Education
Program Adult Treatment Panel III (ATP III) ${ }^{13}$, uses five variables for diagnosis of the syndrome, including central obesity, high triglycerides, low high-density lipoprotein (HDL) cholesterol, high blood pressure and higher fasting glucose. Since the ATP III's definition of central obesity as waist circumference $\geq 102 \mathrm{~cm}$ for men and $88 \mathrm{~cm}$ for women might be inappropriate for Asian populations, some studies suggested modifying the APT III by incorporating the Asian-Pacific waist circumference cut-off $(90 \mathrm{~cm}$ for men and $80 \mathrm{~cm}$ for women $)^{14,15}$. This adjustment has been incorporated into the International Diabetes Federation (IDF) definition of metabolic syndrome ${ }^{16}$. IDF also modified the original ATP III criteria by giving a higher weight for central obesity than other disorders and by incorporating the lowered fasting plasma glucose cut-off as proposed by the American Diabetes Association ${ }^{16}$.

The purpose of this study was to assess the impact of urbanisation on the risk of chronic diseases, by comparing 
the prevalence of the metabolic syndrome in rural and urban adults in East China.

\section{Subjects and methods}

\section{Subjects}

The China Nutrition Transition study was a communitybased cross-sectional survey performed in 2002. A threestage stratified random sampling method was used to select the study sample, residing in the National Disease Surveillance Points (DSP) in East China. The DSP registry system was established in 1989 and was comprised of 145 nationally representative 'disease surveillance points', across seven geographic regions and 30 provinces of China ${ }^{17}$. In the first stage, we randomly selected two rural regions (Wuning County in Jiangxi Province and Juchao County in Anhui Province) and one urban district (Jing'an District of Shanghai) from $\sim 20$ disease registry points in East China. In the second stage, we selected at random 10 villages or neighbourhoods from each region. The third stage consisted of a random selection of 10 households from each village or neighbourhood based on local household census data. Because we sampled only three sites, the data may not be representative of the whole population of Eastern China, but it is still a valid sample to compare urban-rural differences in that region.

All family members aged 8-64 years were eligible for the study. We excluded pregnant and lactating women, and subjects who had been diagnosed with diabetes and were taking antidiabetic medications. The present report is restricted to adult subjects 20-64 years of age. All subjects were of the Han ethnicity, the most predominant group in China. The participation rate was 78\% in rural and 70\% in urban areas. The main reason for non-participation was absence from the residential area on the day of the examination.

All subjects gave their written, informed consent. The study protocol was approved by the Committee on Human Research of The Johns Hopkins University Bloomberg School of Public Health and by the Chinese Academy of Medical Science.

\section{Measurement}

Participants were asked to fast overnight and to attend a local health care station for a physical exam. Under the supervision of study investigators, data collection was performed by local health care workers after comprehensive training on each specific tool and methodology employed. Weight and height were measured with subjects wearing light clothing and no shoes. Waist circumference was measured at the level of the navel.

Socio-economic status, education, occupation, smoking status, alcohol consumption and physical activity were assessed using Behavior Risk Factor Survey System Questionnaire $^{18}$. The usual amount and frequency of alcohol intake for each kind of alcoholic beverage in the past year were obtained. Since only 24 subjects (4.67\%) in this study population are ex-smokers, we grouped smoking status into current smoker and non-current smoker categories. We dichotomised physical activity into sedentary and non-sedentary categories, based on occupational physical activity, which in the Chinese population accounts for almost $80 \%$ of daily physical activity $^{19}$. Four 24-hour dietary recalls were collected in rural sites, one per season. Three dietary recalls were conducted in the urban site in spring, summer and autumn. All days of the week were equally represented in the collection of dietary recall. A complete set of Chinese food models was displayed to help subjects estimate the amount of food eaten. The 2002 Chinese food composition table was used to calculate macronutrient intake.

Systolic and diastolic blood pressures were measured twice after 5 min rest in the seated position using a mercury sphygmomanometer. First and fifth Korotkoff sounds were taken as systolic and diastolic blood pressure, respectively. All subjects underwent an oral glucose tolerance test following the World Health Organization protocol ${ }^{20}$.

Blood specimens were centrifuged immediately after blood was drawn at each site, and shipped in the frozen state, using dry ice, to a central clinical laboratory in Shanghai No. 6 People's Hospital. Specimens had been stored at $-70^{\circ} \mathrm{C}$ until laboratory assays could be carried out. Plasma glucose was measured in an automated analyser (Hitachi High-Technologies Corporation) using the glucose oxidase method (intra- and inter-assay coefficient of variation $(\mathrm{CV})<5 \%$ ). Triglycerides and HDL cholesterol were measured in the same analyser by an enzymatic method (triglycerides: intra- and inter-assay CV $<5 \%$; HDL cholesterol: intra-assay CV 1.5\%, inter-assay CV 2.1\%) ${ }^{21}$. Plasma insulin was determined by radioimmunoassay (intraand inter-assay CV < 10\%) (Linco Research, Inc.).

\section{Definition of the metabolic syndrome}

The metabolic syndrome was defined according to the ATP III definition ${ }^{13}$ as the presence of three or more of the following criteria: central obesity as waist circumference $\geq 102 \mathrm{~cm}$ (men) and $88 \mathrm{~cm}$ (women); plasma triglycerides $\geq 1.7 \mathrm{mmoll}^{-1}$; HDL cholesterol $<1.0 \mathrm{mmoll}^{-1}$ (men) and $1.3 \mathrm{mmoll}^{-1}$ (women); fasting plasma glucose $\geq 6.1 \mathrm{mmoll}^{-1}$; and blood pressure $\geq 135 / 95 \mathrm{mmHg}$. The ATP III criteria are ambiguous about subjects who are being treated for hypertension. We classified subjects using antihypertensive medications as having hypertension. We also modified the ATP III by incorporating the Asian waist circumference cut-off point at $90 \mathrm{~cm}$ for men and $80 \mathrm{~cm}$ for women ${ }^{16,22}$. In addition, we diagnosed metabolic syndrome using the IDF criteria: central obesity ( $\geq 80 \mathrm{~cm}$ for women, $90 \mathrm{~cm}$ for men) plus two of the following: raised triglycerides, reduced HDL cholesterol, raised blood pressure or raised fasting plasma glucose. In contrast to ATP III, IDF used a lower cut-off of $5.6 \mathrm{mmol}^{-1}$ to define raised fasting plasma glucose ${ }^{16}$. 


\section{Statistical analysis}

Statistical analysis was performed sing SAS software, version 8.0 (SAS Institute, Inc.) and STATA, version 7.0 (Stata Corp.). The sampling weights were calculated based on the year 2000 China Population Census data and our sampling scheme, and took into account nonresponse and demographic differences in age and gender between the sample and the total population. The sampling weight and complex sampling design were incorporated into all statistical analyses to derive the weighted mean, percentage and standard error. Natural logarithm transformation was used for rightskewed variables, including fasting and $2 \mathrm{~h}$ glucose and insulin, triglycerides, total, low-density lipoprotein (LDL) and HDL cholesterol and the ratio of LDL to HDL cholesterol. We stratified the analysis by location and gender. Logistic regression models were used to contrast the urban-rural difference with adjustment for age; likewise, linear regression models were used to compare continuously distributed risk factor levels with controlling for age. The prevalence of the metabolic syndrome and its components was age-standardised by a direct method using the 2000 adult population of China (20-64 years) as the standard population.

\section{Results}

\section{Subjects' characteristics}

More than $90 \%$ of our rural subjects were farmers, fishermen or housewives, while the majority of urban residents were civil servants, salesmen, health care workers and industrial labourers. Urban residents had a higher income and education level than their rural counterparts. Smoking prevalence, daily alcohol and energy intake were higher in men than in women, and slightly higher in rural residents than in urban residents. The percentage of dietary fat intake was higher in urban than in rural subjects, especially in women. More than $75 \%$ of urban adults had a sedentary occupational physical activity, as compared with a rate of $<15 \%$ in rural residents (Table 1).

\section{Urban-rural comparison of the metabolic syndrome}

Table 2 compares the age-adjusted mean of various anthropometric and metabolic indices by location in men and women separately. Body mass index (BMI), waist circumference and $2 \mathrm{~h}$ glucose were significantly higher in urban men than in rural men, but the urban-rural difference was not significant in women. Dwelling in urban areas was associated with significantly higher $2 \mathrm{~h}$ plasma insulin, triglycerides, and total and LDL cholesterol in both men and women. In men, HDL cholesterol was higher in rural than urban residents while the urban-rural difference was the opposite in women. As for gender difference in each location, we observed that urban women had significantly lower waist circumference and triglycerides and higher HDL cholesterol than urban men. Conversely, rural women had higher metabolic risk than rural men, as demonstrated by higher BMI, waist circumference, fasting and $2 \mathrm{~h}$ plasma insulin and triglycerides.

Table 3 shows the age-standardised prevalence of the metabolic syndrome and its components. The prevalence of high triglycerides was significantly greater in urban residents than in rural adults. No significant urban-rural difference in the prevalence of high fasting plasma glucose was observed. In men, urban subjects had a higher prevalence of low HDL cholesterol and hypertension, as compared with rural men. In women, the prevalence of low HDL cholesterol was significantly higher in rural areas. According to the original ATP III criteria, very few subjects were centrally obese, especially for men: none among rural men, and 2\% among urban men. The prevalence of the metabolic syndrome was significantly higher for urban men (12.7\%) than for rural men (1.7\%), and was similar between urban women (10.1\%) and rural women (9.7\%).

Using the modified ATP III criteria, the prevalence of central obesity increased by 18 -fold for men and 2.5-fold for women, and the rate was significantly higher in urban subjects than rural subjects in men (39.01 vs. $4.67 \%$, $P<0.001$ ) and women (55.81 vs. $40.76 \%, P=0.02$ ). Also, the prevalence of the metabolic syndrome increased more

Table 1 Selected characteristics of the rural and urban study populations

\begin{tabular}{lcccccc}
\hline & \multicolumn{3}{c}{ Men } & & \multicolumn{3}{c}{ Women } \\
\cline { 2 - 3 } & Rural $(n=177)$ & Urban $(n=81)$ & $P^{*}$ & Rural $(n=191)$ & Urban $(n=80)$ & $P^{*}$ \\
\hline Education less than senior high school (\%) & 94.26 & 17.12 & $<0.001$ & 98.43 & 16.78 & $<0.001$ \\
Self-reported monthly family income $<500 ¥$ & 95.96 & 2.98 & $<0.001$ & 98.78 & 3.41 & $<0.001$ \\
Current smoker (\%) $_{\text {Alcohol intake (g day }}^{-1}$ ) & 74.02 & 50.23 & 0.05 & 1.27 & 0.90 & 0.73 \\
Energy intake (kcal day $^{-1}$ ) & $14.63 \pm 40.17$ & $8.98 \pm 27.54$ & 0.50 & $0.60 \pm 1.93$ & $0.37 \pm 2.59$ & 0.18 \\
Fat intake (\%) & $2600 \pm 984$ & $2500 \pm 864$ & 0.23 & $2059 \pm 621$ & $2001 \pm 688$ & 0.52 \\
Sedentary occupation (\%) & $30.27 \pm 24.48$ & $32.92 \pm 9.90$ & 0.17 & $28.92 \pm 6.50$ & $37.42 \pm 5.37$ & $<0.001$ \\
& 7.89 & 71.92 & $<0.001$ & 13.11 & 82.54 & $<0.001$ \\
\hline
\end{tabular}

Data are mean \pm standard deviation or \%.

${ }^{*} P$-value for urban-rural comparison with adjustment for age in men and women. 
Table 2 Age-adjusted comparison of metabolic indices between locations and between genders

\begin{tabular}{|c|c|c|c|}
\hline & Rural & Urban & $P^{*}$ \\
\hline \multicolumn{4}{|c|}{ BMI $\left(\mathrm{kg} \mathrm{m}^{-2}\right)$} \\
\hline Men & $21.10 \pm 4.39$ & $23.85 \pm 3.78$ & $<0.001$ \\
\hline Women & $22.36 \pm 5.94$ & $22.62 \pm 2.95$ & 0.69 \\
\hline$P$-value $\dagger$ & 0.014 & 0.06 & \\
\hline \multicolumn{4}{|c|}{ Waist circumference $(\mathrm{cm})$} \\
\hline Men & $75.37 \pm 11.04$ & $86.02 \pm 13.68$ & $<0.001$ \\
\hline Women & $79.07 \pm 12.85$ & $81.21 \pm 9.66$ & 0.21 \\
\hline$P$-value $\dagger$ & 0.001 & 0.025 & \\
\hline \multicolumn{4}{|c|}{ Fasting plasma glucose $\left(\mathrm{mmol} \mathrm{I}^{-1}\right) \ddagger$} \\
\hline Men & $5.00 \pm 1.33$ & $5.03 \pm 0.81$ & 0.93 \\
\hline Women & $5.05 \pm 1.38$ & $4.81 \pm 0.63$ & 0.04 \\
\hline$P$-value $\dagger$ & 0.23 & 0.013 & \\
\hline \multicolumn{4}{|c|}{$2 \mathrm{~h}$ plasma glucose $\left(\mathrm{mmol} \mathrm{I}^{-1}\right) \ddagger$} \\
\hline Men & $5.47 \pm 2.39$ & $6.55 \pm 2.61$ & 0.003 \\
\hline Women & $5.81 \pm 1.80$ & $5.96 \pm 1.97$ & 0.68 \\
\hline$P$-value $\dagger$ & 0.09 & 0.06 & \\
\hline \multicolumn{4}{|c|}{ Fasting plasma insulin $\left(\mu \cup \mathrm{ml}^{-1}\right) \ddagger$} \\
\hline Men & $7.77 \pm 9.05$ & $8.67 \pm 8.91$ & 0.41 \\
\hline Women & $9.39 \pm 5.80$ & $8.41 \pm 7.60$ & 0.31 \\
\hline$P$-value $\dagger$ & 0.03 & 0.71 & \\
\hline \multicolumn{4}{|c|}{$2 \mathrm{~h}$ plasma insulin $\left(\mu \mathrm{U} \mathrm{ml} \mathrm{l}^{-1}\right) \ddagger$} \\
\hline Men & $19.20 \pm 19.42$ & $42.53 \pm 50.22$ & $<0.001$ \\
\hline Women & $30.72 \pm 29.42$ & $47.46 \pm 33.54$ & $<0.001$ \\
\hline$P$-value $\dagger$ & $<0.001$ & 0.78 & \\
\hline \multicolumn{4}{|c|}{ Triglycerides $\left(\mathrm{mmol} \mathrm{I}^{-1}\right) \ddagger$} \\
\hline Men & $0.65 \pm 0.67$ & $1.52 \pm 0.99$ & $<0.001$ \\
\hline Women & $0.78 \pm 0.41$ & $1.04 \pm 0.80$ & 0.006 \\
\hline$P$-value $\dagger$ & 0.003 & 0.003 & \\
\hline \multicolumn{4}{|c|}{ Total cholesterol $\left(\mathrm{mmol} \mathrm{I}^{-1}\right) \ddagger$} \\
\hline Men & $3.39 \pm 1.73$ & $4.22 \pm 0.72$ & $<0.001$ \\
\hline Women & $3.39 \pm 1.11$ & $4.45 \pm 0.72$ & $<0.001$ \\
\hline$P$-value $\dagger$ & 0.95 & 0.05 & \\
\hline \multicolumn{4}{|c|}{ LDL cholesterol $\left(\mathrm{mmol} \mathrm{I}^{-1}\right) \ddagger$} \\
\hline Men & $1.98 \pm 1.60$ & $2.73 \pm 0.72$ & $<0.001$ \\
\hline Women & $1.99 \pm 0.83$ & $2.73 \pm 0.89$ & $<0.001$ \\
\hline \multirow{2}{*}{\multicolumn{4}{|c|}{ HDL cholesterol $\left(\mathrm{mmol} \mathrm{I}^{-1}\right) \ddagger$}} \\
\hline & & & \\
\hline Men & $1.22 \pm 0.40$ & $1.09 \pm 0.18$ & 0.001 \\
\hline Women & $1.20 \pm 0.41$ & $1.42 \pm 0.54$ & 0.001 \\
\hline$P$-value & 0.27 & $<0.001$ & \\
\hline \multicolumn{4}{|c|}{ LDL to HDL ratio $\ddagger$} \\
\hline Men & $1.62 \pm 1.20$ & $2.51 \pm 0.90$ & $<0.001$ \\
\hline Women & $1.66 \pm 0.55$ & $1.91 \pm 1.16$ & 0.09 \\
\hline$P$-value $\dagger$ & 0.59 & 0.001 & \\
\hline
\end{tabular}

BMI - body mass index; LDL - low-density lipoprotein; HDL - high-density lipoprotein.

Data are mean \pm standard deviation.

${ }^{*} P$-value for comparison between sites in men and women respectively. $\dagger P$-value for comparison between gender at each site.

$\ddagger$ Geometric mean and standard error are given.

in urban subjects than in rural subjects, and we observed a significant urban-rural difference in the metabolic syndrome prevalence in both men (34.3 vs. 2.7\%, $P<0.01)$ and women (24.1 vs. $11.4 \%, P=0.07)$. The prevalence estimated using IDF's definition was very close to that using the modified ATP III criteria, and showed a significant urban-rural difference in men (30.49 vs. 2.25, $P<0.01)$, and a less evident difference in women (25.01 vs. $14.58, P=0.18)$. Regardless of whether the original or modified ATP III criteria or IDF's definition was used, our logistic regression analysis demonstrated a significant interaction between gender and residency for the odds ratio of having the metabolic syndrome $(P<0.01)$

\section{Sensitivity and specificity analysis of waist circumference cut-offs}

Using receiver operating characteristic analysis, we compared the capability of waist circumference cut-offs of the APT III and Asian criteria in identifying at least two other features of the metabolic syndrome, which included high glucose, high blood pressure, high triglycerides and low HDL cholesterol. The Asian cut-off had a better combination of sensitivity (0.64) and specificity (0.72) than those of the ATP III (sensitivity 0.23, specificity 0.93). Also, the area under curve for Asian cut-offs was significantly higher than those of the ATP III (0.69 vs. 0.58, $P<0.001)$ (Fig. 1)

\section{Discussion}

In the present study, we assessed urbanisation's impact on the risk of chronic diseases by comparing urban-rural differences in the prevalence of the metabolic syndrome. As defined by the ATP criteria, we found a significantly higher prevalence of the syndrome in urban than in rural men (12.7 vs. $1.7 \%, P<0.01)$ and essentially no difference among women (10.1 vs. 9.7\%, $P=0.17$ ). The crude prevalence for the whole population sample was $\sim 7 \%$, which was comparable with the rate of $10 \%$ in Chinese aged 30-74 years ${ }^{23}$. When we modified the ATP III criteria on the basis of recommendations for Asian populations, the urban-rural differences became more pronounced for both genders: 34.3 vs. $2.7 \%(P<0.01)$ in men, and 24.1 vs. $11.4 \%(P=0.07)$ in women. Using the most recent definition proposed by the IDF, the prevalence rate is similar to that using the modified APT III criteria.

In the present study, we excluded subjects with a selfreported history of diagnosed diabetes; however, a recent national survey has shown that only $2-3 \%$ of urban adults and $1 \%$ of rural adults had such self-reported history ${ }^{24}$. Therefore, this exclusion should make our prevalence only slightly lower than that in the general population. As shown by our data, the prevalence of the metabolic syndrome in China is much lower than in the USA ${ }^{25}$, but is comparable with that from other Asian populations using the ATP III criteria. A study in Taiwanese adults reported a prevalence of $9.5 \%{ }^{26}$, and other studies have reported prevalences of $6-14 \%$ in Hong Kong residents ${ }^{27,28}$. A recent study in urban Koreans aged 30-80 years showed that 16\% of men and $10 \%$ of women had the syndrome ${ }^{14}$. A cross-sectional study in Singapore that included 18-69 year olds, showed a prevalence of $12 \%$ for all races and of $9.4 \%$ for Chinese ${ }^{15}$. However, the direct comparison must be made with caution since the selection of the samples was different, for example the age distribution and proportion of urban to rural populations, and the time of the study, which affect the prevalence estimate.

Using either the original or the modified ATP III criteria, our results showed that urbanisation's impact on the prevalence of metabolic syndrome was significantly modified by gender: the urban-rural difference was 
Table 3 Age-standardised prevalence of the metabolic syndrome in the rural and urban men (M) and women (W)

\begin{tabular}{|c|c|c|c|c|c|c|}
\hline & \multicolumn{2}{|c|}{ Men } & \multirow[b]{2}{*}{$P^{*}$} & \multicolumn{2}{|c|}{ Women } & \multirow[b]{2}{*}{$P^{*}$} \\
\hline & $\begin{array}{c}\text { Rural } \\
(n=177)\end{array}$ & $\begin{array}{l}\text { Urban } \\
(n=81)\end{array}$ & & $\begin{array}{c}\text { Rural } \\
(n=191)\end{array}$ & $\begin{array}{c}\text { Urban } \\
(n=80)\end{array}$ & \\
\hline Triglycerides $\geq 1.7 \mathrm{mmol} \mathrm{I}^{-1}$ & 2.73 & 41.78 & $<0.001$ & 4.99 & 18.06 & 0.02 \\
\hline $\mathrm{HDL}$ cholesterol $<1.0 \mathrm{mmol} \mathrm{I}^{-1}(\mathrm{M}),<1.3 \mathrm{mmol} \mathrm{I}^{-1}(\mathrm{~W})$ & 20.03 & 33.41 & 0.15 & 59.22 & 39.01 & 0.04 \\
\hline Blood pressure $\geq 130 / 85 \mathrm{mmHg}$ or using medication & 24.05 & 38.74 & 0.22 & 14.49 & 15.97 & 0.73 \\
\hline Fasting plasma glucose $\geq 6.1 \mathrm{mmol} \mathrm{I}^{-1}$ & 4.93 & 2.65 & 0.29 & 4.40 & 2.95 & 0.54 \\
\hline Fasting plasma glucose $\geq 5.6 \mathrm{mmol} \mathrm{I}^{-1}$ & 23.11 & 9.47 & 0.25 & 26.25 & 5.51 & 0.02 \\
\hline Waist circumference $\geq 102 \mathrm{~cm}(\mathrm{M}), 88 \mathrm{~cm}(\mathrm{~W})$ & 0.00 & 2.47 & 0.19 & 18.39 & 17.70 & 0.92 \\
\hline Waist circumference $\geq 90 \mathrm{~cm}(\mathrm{M}), 80 \mathrm{~cm}(\mathrm{~W})$ & 4.67 & 39.01 & $<0.001$ & 40.76 & 55.81 & 0.02 \\
\hline ATP III metabolic syndrome & 1.66 & 12.65 & 0.009 & 9.67 & 10.12 & 0.17 \\
\hline Modified ATP III metabolic syndrome & 2.72 & 34.26 & $<0.001$ & 11.40 & 24.07 & 0.07 \\
\hline IDF metabolic syndrome & 2.25 & 30.49 & $<0.001$ & 14.58 & 25.01 & 0.18 \\
\hline
\end{tabular}

HDL - high-density lipoprotein; ATP III - Adult Treatment Panel III; IDF - International Diabetes Foundation.

Data are the percentage and prevalence age-standardized by using the year 2000 Chinese population aged 20-64 years.

${ }^{*} P$-value for urban-rural difference with adjustment for age in men and women.

much more evident in men than in women. Previous studies have reported a similar pattern for the prevalence of hypertension, diabetes, impaired fasting glucose and the metabolic syndrome $e^{5,23,24}$. We also observed that urban Chinese women had a higher HDL cholesterol level than rural women, consistent with other reports ${ }^{4,23}$.

Our study found that urban dwellers had higher fat intake and higher levels of physical inactivity than their rural counterparts, which was consistent with a previous report ${ }^{2}$. While these differences were similar for men and women, the urban-rural difference in metabolic syndrome prevalence was much more evident for men than for women. In other words, for the metabolic syndrome, rural women were similar to urban women. The reasons for this are unclear. Rural women have a higher fertility rate than urban women $(\geq 2.5 \text { vs. } 1.2)^{1}$. It has been suggested that multiple pregnancies may increase the risk of developing central obesity, insulin resistance and hypertension ${ }^{29,30}$, all

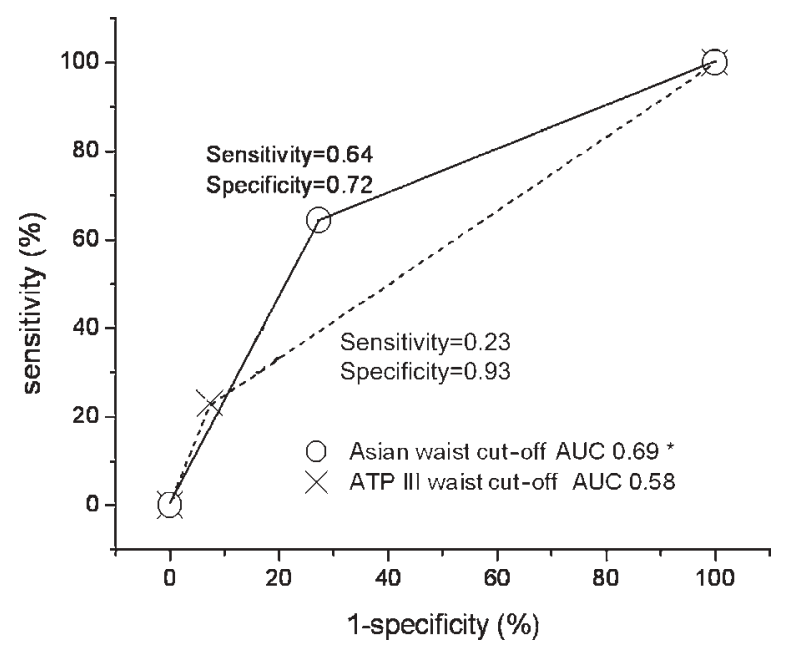

Fig. 1 Sensitivity and specificity of waist circumference cut-off for Asians and the Adult Treatment Panel III (ATP III) in identifying metabolic disorders. ${ }^{*}$ The area under the curve (AUC) of the Asian waist cut-off is significantly higher than that of the ATP III $\left(\chi^{2}=16.68, \mathrm{df}=1, P<0.001\right)$ components of the metabolic syndrome. If so, the favourable effects of rural dwelling on metabolic indices may be diminished by the unfavourable effects of the higher fertility rate of rural women.

Another interesting finding is the opposite gender effect on the metabolic syndrome in rural and urban areas: in the urban environment women had a lower risk than men, whereas the opposite was true in rural areas. Like our results, studies in Korean population showed the same pattern of difference across gender ${ }^{14,23,31}$. In China, previous studies have shown that urban men have a higher risk of obesity ${ }^{32}$, metabolic syndrome ${ }^{33}$, hypertension ${ }^{5}$, diabetes and impaired fasting glucose $\mathrm{e}^{24}$, and cardiovascular disease $\mathrm{s}^{32}$, as compared with urban women. However, studies in rural China have shown that rural women have a higher prevalence of obesity and have higher triglyceride concentrations than rural men ${ }^{24,34,35}$.

In conclusion, our results indicate a significantly higher prevalence of the metabolic syndrome among urbandwelling Chinese, especially men. In contrast, the prevalence of the metabolic syndrome among rural women was similar to that or urban women.

\section{Acknowledgements}

Sources of funding: This study was funded by the Center of a Livable Future, Johns Hopkins Bloomberg School of Public Health.

Conflict of interest declaration: No author declares a conflict of interest with this work.

Authorship responsibilities: X.W. performed the statistical analysis. The manuscript was drafted by X.W. and B.C. All authors participated in the study design, data collection and interpretation, and reviewed and approved the manuscript.

Acknowledgements: We gratefully acknowledge the valuable assistance of the staff of the regional offices of the Chinese Center for Disease Control and Prevention in Wuning (Jiangxi Province), Chaohu (Anhui Province) and the Jian'an District of Shanghai. 


\section{References}

1 Executive Summary: Demographic Profile of China [article online]. http://www.prcdc.org/summaries/china/china. html, 2002. Accessed 11 October 2003.

2 Caballero B, Popkin BM. The Nutrition Transition: Diet and Disease in the Developing World. London: Academic Press, 2002.

3 He J, Klag MJ, Wu Z, Qian MC, Chen JY, Mo PS, et al. Effect of migration and related environmental changes on serum lipid levels in southwestern Chinese men. American Journal of Epidemiology 1996; 144: 839-48.

4 Woo KS, Chook P, Raitakari OT, McQuillan B, Feng JZ, Celermajer DS. Westernization of Chinese adults and increased subclinical atherosclerosis. Arteriosclerosis, Thrombosis, and Vascular Biology 1999; 19: 2487-93.

5 Reynolds K, Gu D, Muntner P, Wu X, Chen J, Huang G, et al. Geographic variations in the prevalence, awareness, treatment and control of hypertension in China. Journal of Hypertension 2003; 21: 1273-81.

6 Reaven GM. Banting Lecture 1988. Role of insulin resistance in human disease. Diabetes 1988; 37: 1595-607.

7 Haffner SM, Valdez RA, Hazuda HP, Mitchell BD, Morales PA, Stern MP. Prospective analysis of the insulin-resistance syndrome (syndrome X). Diabetes 1992; 41: 715-22.

8 Wang JJ, Hu G, Miettinen ME, Tuomilehto J. The metabolic syndrome and incident diabetes: assessment of four suggested definitions of the metabolic syndrome in a Chinese population with high post-prandial glucose. Hormone and Metabolic Research 2004; 36: 708-15.

9 Wang JJ, Qiao Q, Miettinen ME, Lappalainen J, Hu G, Tuomilehto $\mathrm{J}$. The metabolic syndrome defined by factor analysis and incident type 2 diabetes in a Chinese population with high postprandial glucose. Diabetes Care 2004; 27: 2429-37.

10 Isomaa B, Almgren P, Tuomi T, Forsen B, Lahti K, Nissen M, et al. Cardiovascular morbidity and mortality associated with the metabolic syndrome. Diabetes Care 2001; 24: 683-9.

11 Lakka HM, Laaksonen DE, Lakka TA, Niskanen LK, Kumpusalo E, Tuomilehto J, et al. The metabolic syndrome and total and cardiovascular disease mortality in middleaged men. Journal of the American Medical Association 2002; 288: 2709-16.

12 Hu G, Qiao Q, Tuomilehto J, Balkau B, Borch-Johnsen K, Pyorala K. Prevalence of the metabolic syndrome and its relation to all-cause and cardiovascular mortality in nondiabetic European men and women. Archives of Internal Medicine 2004; 164: 1066-76.

13 Executive Summary of the Third Report of The National Cholesterol Education Program (NCEP). Expert Panel on detection, evaluation, and treatment of high blood cholesterol in adults (Adult Treatment Panel III). Journal of the American Medical Association 2001; 285: 2486-97.

14 Oh JY, Hong YS, Sung YA, Barrett-Connor E. Prevalence and factor analysis of metabolic syndrome in an urban Korean population. Diabetes Care 2004; 27: 2027-32.

15 Tan CE, Ma S, Wai D, Chew SK, Tai ES. Can we apply the National Cholesterol Education Program Adult Treatment Panel definition of the metabolic syndrome to Asians? Diabetes Care 2004; 27: 1182-6.

16 Zimmet P, Magliano D, Matsuzawa Y, Alberti G, Shaw J. The metabolic syndrome: a global public health problem and a new definition. Journal of Atherosclerosis and Thrombosis 2005; 12: 295-300.

17 Yang G, Fan L, Tan J, Qi G, Zhang Y, Samet JM, et al. Smoking in China: findings of the 1996 National Prevalence Survey. Journal of the American Medical Association 1999; 282: $1247-53$
18 Centers for Disease Control and Prevention (CDC). Behavioral Risk Factor Surveillance System Survey Questionnaire. Atlanta, Georgia: US, Department of Health and Human Services, Centers for Disease Control and Prevention, 2000.

19 Yao M, McCrory MA, Ma G, Li Y, Dolnikowski GG, Roberts SB. Energy requirements of urban Chinese adults with manual or sedentary occupations, determined using the doubly labeled water method. European Journal of Clinical Nutrition 2002; 56: 575-84.

20 World Health Organization. Diabetes Mellitus. Report of a WHO Study Group. Geneva: WHO, 1985.

21 National Institutes of Health: National Cholesterol Education Program Laboratory Standardization Panel. Recommendations for Improving Cholesterol Measurement: a Report from the Laboratory Standardization Panel of the National Cholesterol Education Program. Bethesda, MD: National Institutes of Health, 1990.

22 World Health Organization. International Association for the Study of Obesity International Obseity TaskForce. The AsiaPacific Perspective: Redefining Obesity and its Treatment. Sydney: Health Communications, 2000.

23 Gu D, Reynolds K, Wu X, Chen J, Duan X, Reynolds RF, et al. Prevalence of the metabolic syndrome and overweight among adults in China. Lancet 2005; 365: 1398-405.

24 Gu D, Reynolds K, Duan X, Xin X, Chen J, Wu X, et al. Prevalence of diabetes and impaired fasting glucose in the Chinese adult population: International Collaborative Study of Cardiovascular Disease in Asia (InterASIA). Diabetologia 2003; 46: 1190-8.

25 Meigs JB, Wilson PW, Nathan DM, D'Agostino RB Sr, Williams K, Haffner SM. Prevalence and characteristics of the metabolic syndrome in the San Antonio Heart and Framingham Offspring Studies. Diabetes 2003; 52: 2160-7.

26 Chuang SY, Chen CH, Chou P. Prevalence of metabolic syndrome in a large health check-up population in Taiwan. Journal of the Chinese Medical Association 2004; 67: 611-20.

27 Ko GT, Cockram CS, Chow CC, Yeung V, Chan WB, So WY, et al. High prevalence of metabolic syndrome in Hong Kong Chinese - comparison of three diagnostic criteria. Diabetes Research and Clinical Practice 2005; 69: 160-8.

28 Patel A, Huang KC, Janus ED, Gill T, Neal B, Suriyawongpaisal $\mathrm{P}$, et al. Is a single definition of the metabolic syndrome appropriate? A comparative study of the USA and Asia. Atherosclerosis 2006; 184: 225-32.

29 Lasky D, Becerra E, Boto W, Otim M, Ntambi J. Obesity and gender differences in the risk of type 2 diabetes mellitus in Uganda. Nutrition 2002; 18: 417-21.

30 Catalano PM. Obesity and pregnancy - the propagation of a viscious cycle? Journal of Clinical Endocrinology and Metabolism 2003; 88: 3505-6.

31 Kim ES, Han SM, Kim YI, Song KH, Kim MS, Kim WB, et al. Prevalence and clinical characteristics of metabolic syndrome in a rural population of South Korea. Diabetic Medicine 2004; 21: 1141-3.

32 Fu FH, Fung L. The cardiovascular health of residents in selected metropolitan cities in China. Preventive Medicine 2004; 38: 458-67.

33 Yao M, Lichtenstein AH, Roberts SB, Ma G, Gao S, Tucker KL, et al. Relative influence of diet and physical activity on cardiovascular risk factors in urban Chinese adults. International Journal of Obesity 2003; 27: 920-32.

34 Hu FB, Chen C, Wang B, Stampfer MJ, Xu X. Leptin concentrations in relation to overall adiposity, fat distribution, and blood pressure in a rural Chinese population. International Journal of Obesity 2001; 25: 121-5.

35 Hu FB, Wang B, Chen C, Jin Y, Yang J, Stampfer MJ, et al. Body mass index and cardiovascular risk factors in a rural Chinese population. American Journal of Epidemiology 2000; 151: 88-97. 